\title{
Estudo e Modelo Estocástico do Algoritmo LMS Restrito ao Ruído de Medição
}

\author{
José Gil F. Zipf, Orlando J. Tobias e Rui Seara
}

\begin{abstract}
Resumo-Este artigo apresenta um modelo estocástico do comportamento médio dos coeficientes e da curva de aprendizagem para o algoritmo LMS restrito ao ruído de medição (NCLMS). O modelo proposto é mais simples do que um outro recentemente apresentado na literatura. A principal característica desse algoritmo é que ele leva em conta a variância do rú́do aditivo no processo de minimização do erro quadrático médio. Como resultado, são incluídos no algoritmo adaptativo alguns parâmetros de controle adicional, melhorando sensivelmente o seu comportamento. Para o controle da estabilidade do algoritmo, algumas indicações úteis de ajuste de tais parâmetros são também fornecidas. Através de simulações numéricas, a precisão do modelo proposto é verificada.
\end{abstract}

Palavras-chave-Modelo estocástico, Comportamento médio do vetor de coeficientes, Curva de aprendizagem, Algoritmo LMS restrito ao ruído, Filtros adaptativos.

Abstract-This paper presents a stochastic model of the mean weight behavior and the learning curve for the noise constrained LMS (NCLMS) algorithm. The proposed model is simpler than another recently presented in the open literature. The main feature of this algorithm is that it takes into account the additive noise variance in the mean-square error (MSE) minimization process. As a result, some additional control parameters are included in the adaptive algorithm, improving the convergence characteristics of the algorithm. Thus, some hints regarding these parameter settings for algorithm stability are also given. Through numerical simulations the accuracy of the proposed model is confirmed.

Keywords-Stochastic model, Mean weight behavior, Learning curve, Noise constrained LMS algorithm, Adaptive filters.

\section{INTRODUÇÃO}

As principais vantagens dos algoritmos adaptativos baseados no gradiente estocástico, como o LMS, são a sua simplicidade e o fato de não necessitarem de conhecimento prévio a respeito das estatísticas do processo [1]. Tais vantagens fazem do algoritmo LMS um dos mais utilizados

José Gil F. Zipf e Rui Seara, LINSE-Laboratório de Circuitos e Processamento de Sinais, Depto. de Eng. Elétrica, Universidade Federal de Santa Catarina, Florianópolis, SC, E-mails: \{gil, seara\}@linse.ufsc.br.

Orlando J. Tobias, LINSE/UFSC e Departamento de Engenharia Elétrica e Telecomunicações, Universidade Regional de Blumenau, SC, E-mail: tobias.oj@ieee.org.

Este trabalho foi parcialmente financiado pelo Conselho Nacional de Desenvolvimento Científico e Tecnológico (CNPq). em aplicações de filtragem adaptativa. Na implementação do algoritmo LMS convencional, o valor do passo de adaptação é fixo; contudo, algoritmos LMS de passo variável (VSLMS) vêm sendo propostos com sucesso. O objetivo de se considerar um passo de adaptação variável é melhorar o desempenho do LMS convencional, principalmente, no que diz respeito à obtenção simultânea de uma convergência rápida com um desajuste reduzido [2], [3]. Recentemente em [4], um novo algoritmo da classe dos VSLMS foi proposto objetivando melhorar o desempenho do LMS convencional em relação ao ruído de medição. Esse algoritmo é denominado algoritmo LMS restrito ao ruído de medição (NCLMS) [4]. O algoritmo NCLMS pode ser eficazmente utilizado em problemas de identificação de sistemas, envolvendo canais com resposta ao impulso finita (FIR) afetados por ruído aditivo Gaussiano. Por exemplo, em comunicações sem fio pode ocorrer uma combinação de desvanecimento e interferência intersimbólica (ISI); assim, um método eficiente de identificação deve ser utilizado com objetivo de acompanhar as rápidas variações do sinal [4]. Realizando medições durante os intervalos de silêncio, é possível obter algum conhecimento da variância do ruído presente no canal. A idéia básica do algoritmo NCLMS é usar tal conhecimento para melhorar o desempenho do algoritmo adaptativo [4], [5].

Este artigo contribui com algumas reflexões no que concerne ao algoritmo NCLMS. Especificamente, apresenta-se um modelo estocástico para o comportamento médio do vetor de coeficientes e para a curva de aprendizagem do algoritmo em questão. O modelo analítico aqui proposto é mais simples do que o apresentado em [4], uma vez que é baseado apenas no momento de primeira ordem. Algumas questões relacionadas à estabilidade e ajuste dos parâmetros do algoritmo NCLMS são também investigadas. Além disso, através de resultados de simulação, verifica-se o desempenho do algoritmo frente a variações de ruído aditivo decorrentes de uma estimação imperfeita. O artigo ainda apresenta uma análise do comportamento do algoritmo em regime permanente.

\section{ALgORITMO NCLMS}

Nesta seção, é brevemente descrito o algoritmo NCLMS [4]. Para tal, considere-se um problema de identificação de sistemas, ilustrado na Fig. 1. 


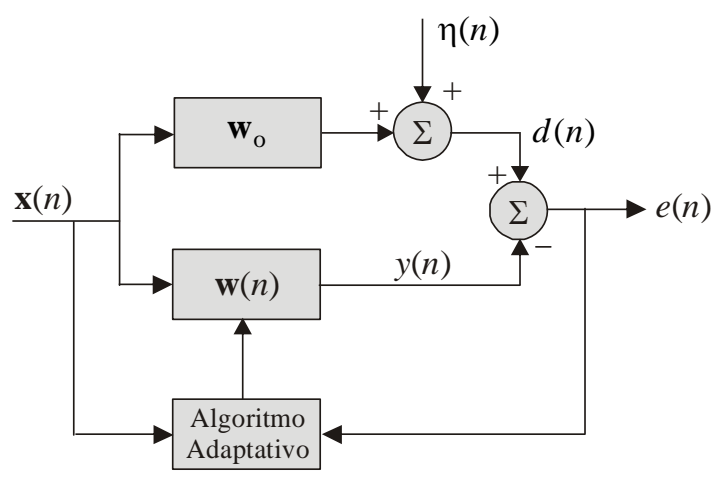

Fig. 1. Diagrama de blocos para identificação de sistemas.

Então, seja a saída de um canal de comunicações dada por

$$
d(n)=\mathbf{w}_{\mathrm{o}}^{\mathrm{T}} \mathbf{x}(n)+\eta(n)
$$

onde o vetor de entrada é $\mathbf{x}(n)=[x(n) x(n-1) \cdots$ $x(n-N+1)]^{\mathrm{T}}$, com $\{x(n)\}$ representando um processo Gaussiano de média zero e variância $\sigma_{x}^{2}, \eta(n)$ é um ruído de medição de média zero e variância $\sigma_{\eta}^{2}$, descorrelacionado de qualquer outro sinal no sistema. O erro quadrático médio (EQM) do processo é

$$
\varepsilon(\mathbf{w})=E\left[e^{2}(n)\right]=E\left\{\left[d(n)-\mathbf{w}^{\mathrm{T}} \mathbf{x}(n)\right]^{2}\right\} .
$$

Então, minimizando (2) com respeito ao vetor $\mathbf{w}$, resulta no vetor ótimo $\mathbf{w}_{\mathrm{o}}=\mathbf{R}^{-1} \mathbf{p}$, onde $\mathbf{R}=E\left[\mathbf{x}(n) \mathbf{x}^{\mathrm{T}}(n)\right]$ é a matriz de autocorrelação do sinal de entrada e $\mathbf{p}=E[\mathbf{x}(n) d(n)]$ é o vetor de correlação cruzada entre o sinal desejado e o vetor de entrada. Assumindo o conhecimento de $\sigma_{\eta}^{2}$, no processo de minimização em (2), obtém-se um problema de otimização com restrições pela minimização de $\varepsilon(\mathbf{w})$ sujeito a $\varepsilon(\mathbf{w})=\sigma_{\eta}^{2}$ [4]. Dessa forma, a nova função-custo é agora dada por

$$
\varepsilon_{1}(\mathbf{w}, \lambda)=\varepsilon(\mathbf{w})+\lambda\left[\varepsilon(\mathbf{w})-\sigma_{\eta}^{2}\right]
$$

Os valores críticos de (3) são $\mathbf{w}=\mathbf{w}_{\mathrm{o}}$ com $\lambda$ qualquer. Conforme [4], essa condição poderia levar a problemas de convergência. Para evitar tais problemas, um termo com uma penalidade adicional $\gamma \lambda^{2} \quad(\operatorname{com} \gamma>0)$ é subtraído de (3), resultando na seguinte expressão:

$$
\varepsilon_{2}(\mathbf{w}, \lambda)=\varepsilon(\mathbf{w})+\lambda\left[\varepsilon(\mathbf{w})-\sigma_{\eta}^{2}\right]-\gamma \lambda^{2} .
$$

Agora, os únicos valores críticos de (4) são $\mathbf{w}=\mathbf{w}_{\mathrm{o}}$ e $\lambda=0$. Na minimização de $\varepsilon_{2}(\mathbf{w}, \lambda),(\mathbf{w}, \lambda)=(\mathbf{c}, 0)$ é um ponto de sela de $\varepsilon_{2}(\mathbf{w}, \lambda)$. Usando o algoritmo de Robbins-Monro [6], obtêm-se as seguintes relações recursivas:

$$
\mathbf{w}(n+1)=\mathbf{w}(n)-\alpha_{\mathrm{f}} \frac{\partial \varepsilon_{2}}{\partial \mathbf{w}}
$$

$$
\lambda(n+1)=\lambda(n)+\beta \frac{\partial \varepsilon 2}{\partial \lambda}
$$

onde $\alpha_{\mathrm{f}}$ e $\beta$ são os passos de adaptação do algoritmo NCLMS.

As derivadas parciais em (5) e (6) são determinadas como segue:

$$
\frac{\partial \varepsilon_{2}}{\partial \mathbf{w}}=-2[1+\gamma \lambda(n)] e(n) \mathbf{x}(n)
$$

e

$$
\frac{\partial \varepsilon_{2}}{\partial \lambda}=\gamma\left[e(n)^{2}-\sigma_{\eta}^{2}\right]-2 \gamma \lambda(n) .
$$

Agora, substituindo (7) e (8) em (5) e (6), obtém-se

$$
w(n)=w(n)+2 \alpha_{\mathrm{f}}[1+\gamma \lambda(n)] e(n) \mathbf{x}(n)
$$

$$
\lambda(n+1)=\lambda(n)+\beta \gamma\left[e(n)^{2}-\sigma_{\eta}^{2}\right]-2 \beta \gamma \lambda(n) .
$$

Então, substituindo $\alpha_{\mathrm{f}}$ por $\frac{\alpha_{\mathrm{f}}}{2}$ e $\beta \gamma$ por $\frac{\beta}{2}$, obtém-se o conjunto de expressões do algoritmo NCLMS. Assim,

$$
\begin{gathered}
\alpha(n)=\alpha_{\mathrm{f}}[1+\gamma \lambda(n)] \\
\mathbf{w}(n+1)=\mathbf{w}(n)+\alpha(n) e(n) \mathbf{x}(n)
\end{gathered}
$$

e

$$
\lambda(n+1)=\lambda(n)+\beta\left\{\frac{1}{2}\left[e^{2}(n)-\sigma_{\eta}^{2}\right]-\lambda(n)\right\} .
$$

A variável $\alpha(n)$ é o fator de passo variável do algoritmo LMS. Note que ele é composto de dois termos, um constante $\alpha_{\mathrm{f}}$ e outro variável $\lambda(n)$. Os parâmetros $0<\beta<1$ e $0<\gamma<1$ são constantes de controle do algoritmo.

\section{Modelo Para o Vetor DE COEFICIENTES}

Considerando $\lambda(0)=0$, a expressão (13) pode ser reescrita como

$$
\lambda(n)=\sum_{k=0}^{n-1}(1-\beta)^{n-1-k} \frac{\beta}{2}\left[e^{2}(k)-\sigma_{\eta}^{2}\right] .
$$

Substituindo (14) em (11), obtém-se

$$
\alpha(n)=\alpha_{\mathrm{f}}+\frac{\alpha_{\mathrm{f}} \beta \gamma}{2} \sum_{k=0}^{n-1}(1-\beta)^{n-1-k}\left[e^{2}(k)-\sigma_{\eta}^{2}\right] .
$$

Definindo $\delta=\frac{1}{2} \alpha_{\mathrm{f}} \beta \gamma$, tem-se

$$
\alpha(n)=\alpha_{\mathrm{f}}+\delta \sum_{k=0}^{n-1}(1-\beta)^{n-1-k}\left[e^{2}(k)-\sigma_{\eta}^{2}\right] .
$$

Agora, substituindo (16) em (12), resulta em

$$
\mathbf{w}(n+1)=\mathbf{w}(n)+\left\{\alpha_{\mathrm{f}}+\delta \sum_{k=0}^{n-1}(1-\beta)^{n-1-k}\left[e^{2}(k)-\sigma_{\eta}^{2}\right]\right\} e(n) \mathbf{x}(n) \text {. }
$$

Definindo a parte variável do passo de adaptação como 


$$
\mu(n)=\delta \sum_{k=0}^{n-1}(1-\beta)^{n-1-k}\left[e^{2}(k)-\sigma_{\eta}^{2}\right]
$$

obtém-se a expressão de atualização do vetor de coeficientes

$$
\mathbf{w}(n+1)=\mathbf{w}(n)+\alpha_{\mathrm{f}} e(n) \mathbf{x}(n)+\mu(n) e(n) \mathbf{x}(n) .
$$

Tomando o valor esperado de ambos os lados de (19), tem-se

$$
E[\mathbf{w}(n+1)]=E[\mathbf{w}(n)]+\alpha_{\mathrm{f}} E[e(n) \mathbf{x}(n)]+E[\mu(n) e(n) \mathbf{x}(n)] .
$$

Arranjando os termos de (20), obtém-se

$$
E[\mathbf{w}(n+1)]=\underbrace{E[\mathbf{w}(n)]+\alpha_{\mathrm{f}}\{\mathbf{p}-\mathbf{R} E[\mathbf{w}(n)]\}}_{\text {LMS convencional }}+E[\mu(n) e(n) \mathbf{x}(n)] \text {. }
$$

Note que (21) é idêntica à expressão que descreve o valor médio do vetor de coeficientes para o algoritmo LMS convencional, exceto pelo último termo da direita, o qual pode ser escrito como

$$
\begin{aligned}
E[\mu(n) e(n) \mathbf{x}(n)]= & \delta E\left[\sum_{k=0}^{n-1}(1-\beta)^{n-1-k} e^{2}(k) e(n) \mathbf{x}(n)\right] \\
& -\delta E\left[\sum_{k=0}^{n-1}(1-\beta)^{n-1-k} \sigma_{\eta}^{2} e(n) \mathbf{x}(n)\right] \\
= & E[\mathbf{a}(n)]-E[\mathbf{b}(n)] .
\end{aligned}
$$

Agora, desenvolvendo cada um dos termos de (22), obtêm-se

$$
\begin{aligned}
E[\mathbf{b}(n)] & =\delta \sum_{k=0}^{n-1}(1-\beta)^{n-1-k} \sigma_{\eta}^{2} E[e(n) \mathbf{x}(n)] \\
& =\delta \sum_{k=0}^{n-1}(1-\beta)^{n-1-k} \sigma_{\eta}^{2}\{\mathbf{p}-\mathbf{R} E[\mathbf{w}(n)]\}
\end{aligned}
$$

e

$$
E\{\mathbf{a}(n)\}=\delta E\left\{\sum_{k=0}^{n-1}(1-\beta)^{n-1-k} e^{2}(k) e(n) \mathbf{x}(n)\right\} .
$$

Reescrevendo agora $E[\mathbf{a}(n)]=E[c(n) e(n) \mathbf{x}(n)]$, onde

$$
c(n)=\delta \sum_{k=0}^{n-1}(1-\beta)^{n-1-k} e^{2}(k)
$$

e assumindo que $c(n)$ seja estatisticamente independente de $e(n)$, resulta em

$$
E[c(n) e(n) \mathbf{x}(n)]=E[c(n)] E[e(n) \mathbf{x}(n)] .
$$

Considerando agora o vetor de erro nos coeficientes, dado por

$$
\mathbf{v}(n)=\mathbf{w}(n)-\mathbf{w}_{\mathrm{o}}
$$

e expressando $E\left[e^{2}(k)\right]$ como função de $\mathbf{R}$ e $\mathbf{v}(n)$, obtém-se

$$
E[c(n)]=\delta \sum_{k=0}^{n-1}(1-\beta)^{n-1-k}\left(\sigma_{\eta}^{2}+\operatorname{tr}\left\{\mathbf{R} E\left[\mathbf{v}(k) \mathbf{v}^{\mathrm{T}}(k)\right]\right\}\right) .
$$

Dado que

$$
E[e(n) \mathbf{x}(n)]=\mathbf{p}-\mathbf{R} E[\mathbf{w}(n)]
$$

e substituindo (28) e (29) em (26), tem-se

$$
\begin{aligned}
& E[c(n)] E[e(n) \mathbf{x}(n)]=\delta \sum_{k=0}^{n-1}(1-\beta)^{n-1-k} \sigma_{\eta}^{2}\{\mathbf{p}-\mathbf{R} E[\mathbf{w}(n)]\} \\
& +\delta \sum_{k=0}^{n-1}(1-\beta)^{n-1-k} \operatorname{tr}\left\{\mathbf{R} E\left[\mathbf{v}(k) \mathbf{v}^{\mathrm{T}}(k)\right]\right\}\{\mathbf{p}-\mathbf{R} E[\mathbf{w}(n)]\} .
\end{aligned}
$$

O primeiro termo no lado direito de (30) é igual a $E[\mathbf{b}(n)]$, dado por (22). Então, substituindo (24) e (30) em (22), obtém-se

$$
\begin{aligned}
E[\mu(n) e(n) \mathbf{x}(n)]=\delta \sum_{k=0}^{n-1}(1-\beta)^{n-1-k} & \operatorname{tr}\left\{\mathbf{R} E\left[\mathbf{v}(k) \mathbf{v}^{\mathrm{T}}(k)\right]\right\} \\
\times & \{\mathbf{p}-\mathbf{R} E[\mathbf{w}(n)]\}
\end{aligned}
$$

Finalmente, de (31), a expressão para o valor médio do vetor de coeficientes é

$$
\begin{aligned}
& E[\mathbf{w}(n+1)]=E[\mathbf{w}(n)]+\alpha_{\mathrm{f}}\{\mathbf{p}-\mathbf{R} E[\mathbf{w}(n)]\} \\
& +\delta \sum_{k=0}^{n-1}(1-\beta)^{n-1-k} \operatorname{tr}\left\{\mathbf{R} E\left[\mathbf{v}(k) \mathbf{v}^{\mathrm{T}}(k)\right]\right\}\{\mathbf{p}-\mathbf{R} E[\mathbf{w}(n)]\} .
\end{aligned}
$$

Nota-se que (32) depende do momento de segunda ordem de w(n) [4]. Contudo, para tal, pode considerar a seguinte aproximação [7]:

$$
E\left[\mathbf{v}(n) \mathbf{v}^{\mathrm{T}}(n)\right] \approx E[\mathbf{v}(n)] E\left[\mathbf{v}^{\mathrm{T}}(n)\right] .
$$

Um modelo simplificado de primeira ordem pode ser obtido por

$$
\begin{aligned}
& E[\mathbf{w}(n+1)]=E[\mathbf{w}(n)]+\alpha_{\mathrm{f}}\{\mathbf{p}-\mathbf{R} E[\mathbf{w}(n)]\} \\
& +\delta \sum_{k=0}^{n-1}(1-\beta)^{n-1-k} \\
& \operatorname{tr}\left(\mathbf{R}\left\{E[\mathbf{w}(k)]-\mathbf{w}_{o}\right\}\left\{E[\mathbf{w}(k)]-\mathbf{w}_{o}\right\}^{\mathrm{T}}\right) \\
& \times\{\mathbf{p}-\mathbf{R} E[\mathbf{w}(n)]\} .
\end{aligned}
$$

\section{Curva de Aprendizagem}

Nesta seção, deriva-se o modelo estatístico para a curva de aprendizagem do algoritmo NCLMS, esta última é definida como

$$
J(n)=\sigma_{\eta}^{2}+\operatorname{tr}[\mathbf{R K}(n)]
$$

para $\quad J(n)=E\left[e^{2}(n)\right] \quad$ e $\quad \operatorname{com} \quad \mathbf{K}(n)=E\left[\mathbf{v}(n) \mathbf{v}^{\mathrm{T}}(n)\right]$ denotando a matriz de covariância do vetor de erro nos coeficientes. Expressando (19) em termos do vetor de erro nos coeficientes, determinado o produto $\mathbf{v}(n) \mathbf{v}^{\mathrm{T}}(n)$, tomando o valor esperado de ambos os lados da expressão resultante e considerando $\mu(n)$ independente de $\mathbf{x}(n)$ e $\mathbf{v}(n)$, obtém-se

$$
\begin{aligned}
\mathbf{K}(n+1) & =\mathbf{K}(n)-E\left[\alpha_{\mathrm{f}}+\mu(n)\right][\mathbf{R K}(n)+\mathbf{K}(n) \mathbf{R}] \\
& +E\left\{\left[\alpha_{\mathrm{f}}+\mu(n)\right]^{2}\right\}\{\mathbf{R} \operatorname{tr}[\mathbf{R K}(n)]+2 \mathbf{R K}(n) \mathbf{R}\} \\
& +E\left\{\left[\alpha_{\mathrm{f}}+\mu(n)\right]^{2}\right\} \mathbf{R} \sigma_{\eta}^{2}
\end{aligned}
$$

Finalmente, a expressão procurada é determinada escrevendo (36) como 


$$
\begin{aligned}
& \mathbf{K}(n+1)=\mathbf{K}(n)-\left\{\alpha_{\mathrm{f}}+E[\mu(n)]\right\}[\mathbf{R K}(n)+\mathbf{K}(n) \mathbf{R}] \\
& +\left\{\alpha_{\mathrm{f}}^{2}+2 \alpha_{\mathrm{f}} E[\mu(n)]+E\left[\mu^{2}(n)\right]\right\}\{2 \mathbf{R K}(n) \mathbf{R}+\mathbf{R} \operatorname{tr}[\mathbf{R K}(n)]\} \\
& +\left\{\alpha_{\mathrm{f}}^{2}+2 \alpha_{\mathrm{f}} E[\mu(n)]+E\left[\mu^{2}(n)\right]\right\} \mathbf{R} \sigma_{\eta}^{2}
\end{aligned}
$$

com

$$
E[\mu(n)]=\delta \sum_{k=0}^{n-1}(1-\beta)^{n-1-k}\left[J(k)-\sigma_{\eta}^{2}\right]
$$

e

$$
E\left[\mu^{2}(n)\right]=\delta^{2} \sum_{k=0}^{n-1}(1-\beta)^{2(n-1-k)}\left[J^{2}(k)-2 J(k) \sigma_{\eta}^{2}+\sigma_{\eta}^{4}\right] .
$$

\section{ANÁLise EM Regime PeRmanente}

Nesta seção, é realizada uma análise do valor de regime para o vetor $\mathbf{w}(n)$, passo variável $\alpha(n)$ e EQM.

\section{A. Vetor $\mathbf{w}(n)$}

Considerando agora que o vetor de coeficientes $\mathbf{w}(n)$ convergiu e utilizando (28) para $n \rightarrow \infty$, tem-se

$$
\begin{aligned}
\mathbf{w}(\infty) & =\mathbf{w}(\infty)+\alpha_{\mathrm{f}}[\mathbf{p}-\mathbf{R w}(\infty)]+\delta \sum_{k=0}^{n-1}(1-\beta)^{n-1-k} \\
& \times J_{\mathrm{ex}}(\infty)[\mathbf{p}-\mathbf{R w}(\infty)]
\end{aligned}
$$

Calculando a soma da série geométrica $\sum_{k=0}^{n-1}(1-\beta)^{n-1-k}=\frac{1}{\beta}$, quando $n \rightarrow \infty$ e com $0<\beta<1$, (40) resulta em

$$
\mathbf{w}(\infty)=\mathbf{w}(\infty)+\alpha_{\mathrm{f}}[\mathbf{p}-\mathbf{R w}(\infty)]+\frac{\delta}{\beta} J_{\mathrm{ex}}(\infty)[\mathbf{p}-\mathbf{R} \mathbf{w}(\infty)]
$$

Fazendo agora $\delta=\frac{1}{2} \alpha_{\mathrm{f}} \beta \gamma$, após algumas manipulações, tem-se

$$
\left[\alpha_{\mathrm{f}}+\frac{1}{2} \alpha_{\mathrm{f}} \gamma J_{\mathrm{ex}}(\infty)\right][\mathbf{p}-\mathbf{R w}(\infty)]=0 .
$$

Visto que $\alpha_{\mathrm{f}}, \gamma$ e $J_{\text {ex }}(\infty)$ são constantes positivas, a única solução de (42) é obtida para

$$
[\mathbf{p}-\mathbf{R w}(\infty)]=0
$$

resultando em

$$
\mathbf{w}(\infty)=\mathbf{R}^{-1} \mathbf{p} .
$$

A partir de (44), constata-se que o valor de regime permanente do algoritmo NCLMS é o mesmo que o do algoritmo LMS convencional.

\section{B. Passo Variável $\alpha(n)$}

Determinando o valor de (11) para $n \rightarrow \infty$, tem-se

$$
\alpha(\infty)=\alpha_{\mathrm{f}}+\frac{1}{2} \alpha_{\mathrm{f}} \gamma J_{\mathrm{ex}}(\infty)
$$

\section{Valor do EQM em Regime Permanente}

Como em regime permanente o passo de adaptação converge para um valor constante, utiliza-se aqui a mesma relação do LMS convencional para um valor de passo de adaptação pequeno. Assim,

$$
J_{\mathrm{ex}}(\infty)=\frac{\mu J_{\min }}{2} \sum_{k=1}^{M} \lambda_{k}
$$

onde $\mu$ é o parâmetro de passo de adaptação constante. Substituindo $\mu$ por $\alpha(\infty)$, tem-se

$$
J_{\mathrm{ex}}(\infty)=\frac{\alpha(\infty) J_{\min }}{2} \sum_{k=1}^{M} \lambda_{k}=\frac{\left[2 \alpha_{\mathrm{f}}+\alpha_{\mathrm{f}} \gamma J_{\mathrm{ex}}(\infty)\right]}{4} J_{\min } \operatorname{tr}(\mathbf{R})
$$

resultando finalmente em

$$
J_{\mathrm{ex}}(\infty)=\frac{2 \alpha_{\mathrm{f}} \sigma_{\eta}^{2} \operatorname{tr}(\mathbf{R})}{4-\alpha_{\mathrm{f}} \gamma \sigma_{\eta}^{2} \operatorname{tr}(\mathbf{R})} .
$$

\section{ROBUSTEZ E ESTABILIDADE}

Dois pontos cruciais relacionados com a aplicação prática do algoritmo NCLMS são observados: a sua robustez com respeito a variações na variância do ruído de medição e os valores máximos (que mantenham a estabilidade do algoritmo) para os parâmetros $\alpha_{\mathrm{f}}, \beta$ e $\gamma$. Na formulação do algoritmo NCLMS, é considerado que a variância do ruído $\sigma_{\eta}^{2}$ seja constante. Contudo, na prática, ela pode ser variante no tempo ou mesmo pode ocorrer um erro em sua estimativa. Na Fig. 2, é ilustrado o desajuste do algoritmo função do erro de estimação da variância do ruído. Dessa figura, nota-se um ligeiro aumento do desajuste do algoritmo frente a grandes variações de $\Delta \sigma_{\eta}^{2} \%$, indicando a robustez do algoritmo em função desse parâmetro.

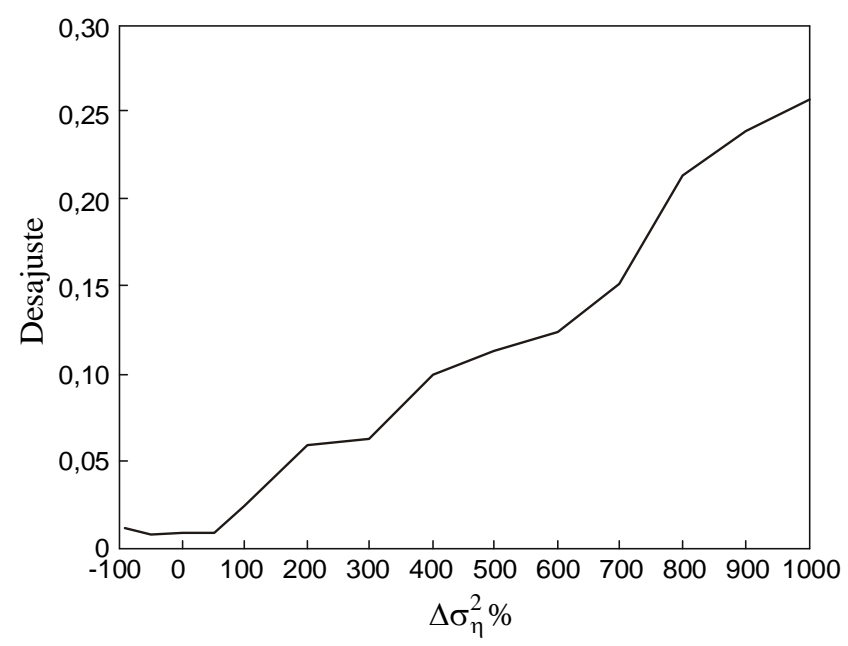

Fig. 2. Desajuste em função de $\Delta \sigma_{\eta}^{2} \%$.

Com relação à estabilidade do algoritmo NCLMS, observa-se que ela está intimamente vinculada com o comportamento dinâmico de $\alpha(n)$. Em geral, a estabilidade dos algoritmos VSLMS está condicionada a uma variação do passo de adaptação confinada aos seguinte limites [8]-[9]:

$$
0<\alpha(n)<\frac{2}{3 \operatorname{tr}[\mathbf{R}]} .
$$


A Fig. 3 apresenta uma curva típica da evolução de $\alpha(n)$. A estabilidade é assegurada se o valor de pico $\alpha_{\max }$ estiver abaixo do valor máximo admissível. Vale lembrar que $\alpha(n)$ depende dos parâmetros $\alpha_{\mathrm{f}}, \beta$ e $\gamma$. Logo eles devem ser escolhidos de forma a garantir um adequado valor de $\alpha_{\max }$. Neste trabalho, é realizada uma investigação heurística do comportamento de $\alpha_{\max }$ em função dos parâmetros $\alpha_{\mathrm{f}}, \beta$ e $\gamma$. Em geral, observa-se, que para filtros de ordem elevada, $\alpha_{\max }$ se mantém constante se os parâmetros $\alpha_{f}, \beta$ e $\gamma$ forem ajustados, considerando que $\delta=0,5 \alpha_{\mathrm{f}} \beta \gamma$ assuma um valor menor do que um valor crítico $\delta_{\text {crit }}$. Além disso, a curva que descreve o comportamento de $\alpha_{\max }$ (em função de $\delta$ ) tem uma forma característica independente da potência média do sinal de entrada $\sigma_{\eta}^{2}$ e da ordem do filtro. Observa-se também que o valor $\delta_{\text {crit }}$, abaixo do qual tem-se a estabilidade do algoritmo, é uma função da potência do sinal de entrada e do comprimento do filtro utilizado. Dessa forma,

$$
\delta<<\delta_{\text {crit }}=f\left(\sigma_{x}^{2}, N\right) .
$$

$\mathrm{Na}$ Fig. 6, são apresentadas algumas curvas do comportamento de $\alpha_{\max }$ em função de $\delta$ como também indicado o valor limite $\delta_{\text {crit }}$.

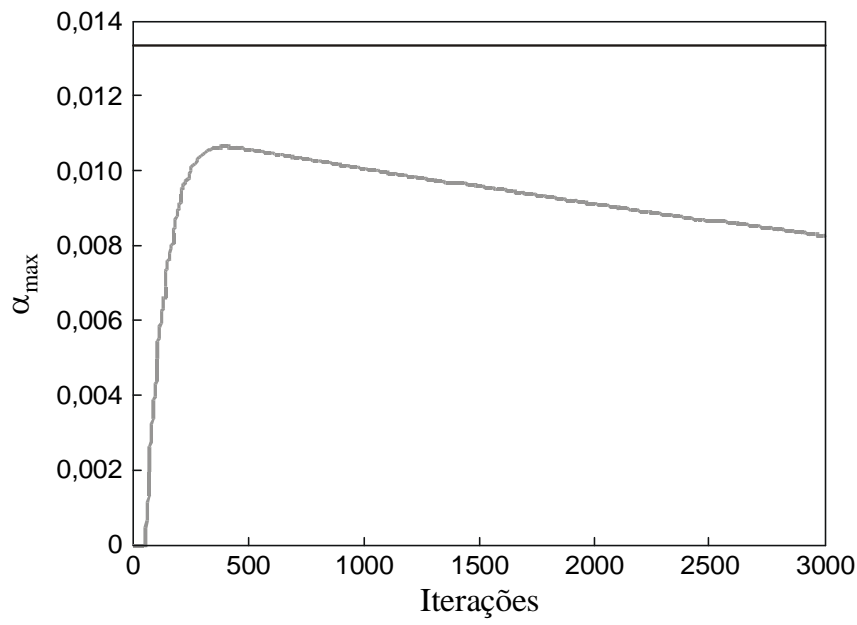

Fig. 3. Evolução de $\alpha(n)$ para um filtro de 50 coeficientes.

\section{Resultados de Simulação}

Nesta seção, são mostrados alguns resultados de simulação numérica verificando a precisão do modelo proposto para o algoritmo NCLMS. Além disso, algumas considerações a respeito da estabilidade do referido algoritmo são apresentadas.

\section{A. Exemplo 1 - Resultados de Modelagem}

Considera-se aqui um problema de identificação de sistemas, utilizando a planta dada por $\mathbf{w}_{0}=[0,2270,460$
$0,6880,3200,110]^{\mathrm{T}}$. Neste exemplo, são considerados ruídos branco e colorido como processos de entradas, sendo em ambos os casos sinais Gaussianos de média zero e variância $\sigma_{x}^{2}=1$. O sinal de entrada correlacionado é obtido por um processo $\mathrm{AR}(1)$, dado por $x(n)=a x(n-1)+u(n)$, onde $a=0,8$ e $u(n)$ é ruído branco tal que $\sigma_{x}^{2}=1$. A variância do ruído de medição é $\sigma_{\eta}^{2}=0,01$. Em ambos os casos, os parâmetros $\alpha_{\mathrm{f}}, \beta$ e $\gamma$ são ajustados conforme [4].

A Fig. 4 mostra os resultados obtidos por simulação Monte Carlo (média de 200 realizações independentes) e pelo modelo proposto. Da Fig. 4, nota-se um bom casamento entre os resultados de simulação e predição. O pequeno desajuste existente na fase transiente [Fig. 4(a)] é devido à aproximação considerada em (33). A Fig. 5 ilustra o caso de sinal de entrada correlacionado. Novamente, um bom casamento entre simulação Monte Carlo (MC) e modelo é verificado.

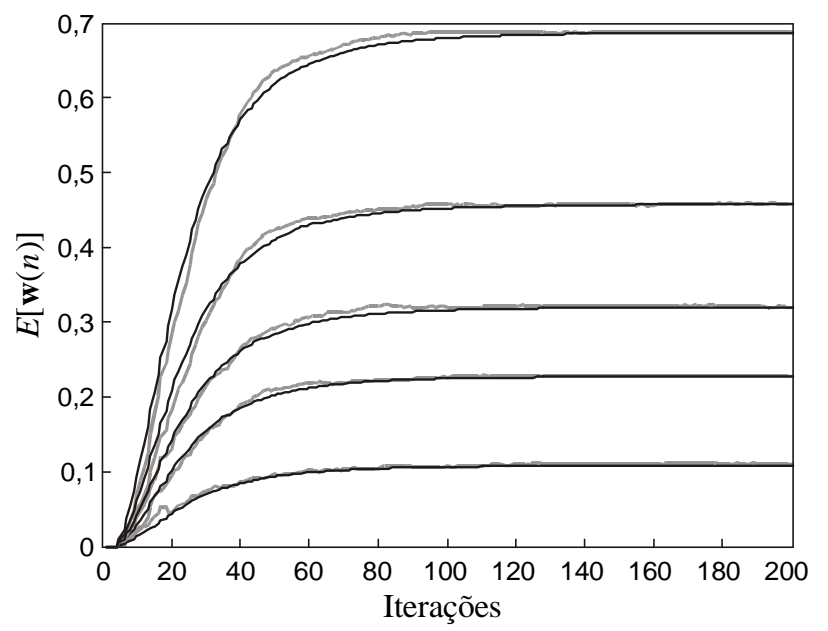

(a)

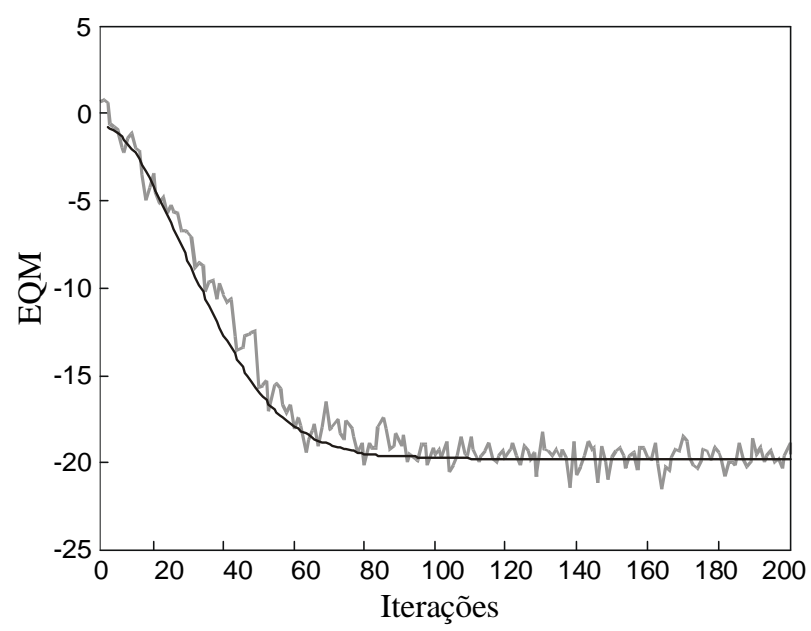

(b)

Fig. 4. Exemplo 1. Sinal de entrada branco. (a) Comportamento médio do vetor de coeficientes: (linha cinza) simulação MC; (linha preta) modelo proposto. (b) Curva de aprendizagem: (linha irregular cinza) simulação MC; (linha contínua preta) modelo proposto. 


\section{B. Exemplo 2 - Considerações de Estabilidade}

Neste exemplo, são apresentadas simulações numéricas para verificar os limites de estabilidade dos diferentes parâmetros do algoritmo, considerando diferentes níveis de ajustes. Além do mais, o desajuste do algoritmo NCLMS sob variações na potência média do ruído de medição é investigado. Para verificação do limite de estabilidade, um filtro de ordem 50 é considerado. A partir de (17) e (18), verifica-se que uma operação estável do algoritmo NCLMS depende dos parâmetros $\alpha_{f}, \beta$ e $\gamma$ através de relações matematicamente complexas. Em particular, a convergência depende de $\alpha_{\mathrm{f}}$ assim como do fator $\delta=0,5 \alpha_{\mathrm{f}} \beta \gamma$. A Fig. 6 mostra uma família de curvas de $\alpha_{\max }$ em função de $\delta$. Cada curva é obtida variando um dos parâmetros, enquanto os outros dois são mantidos fixos. Nota-se dessa figura que todas as curvas obtidas estão fortemente concentradas em torno dos mesmos valores, indicando que para um certo valor de $\delta$ existe aproximadamente um único valor de $\alpha_{\max }$. Observa-se ainda que, para valores de $\delta$ maiores do que $\delta_{\text {crit }}$, o algoritmo não converge.

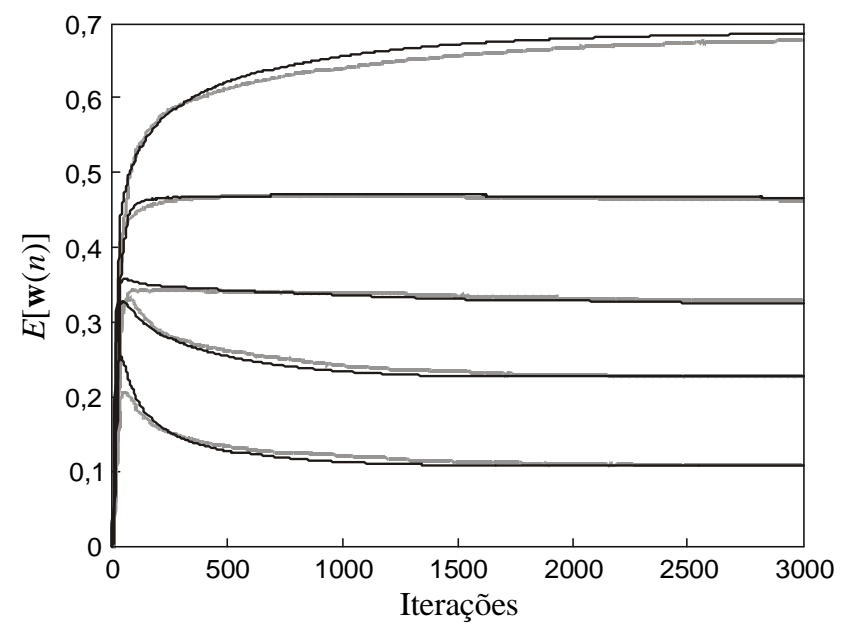

(a)

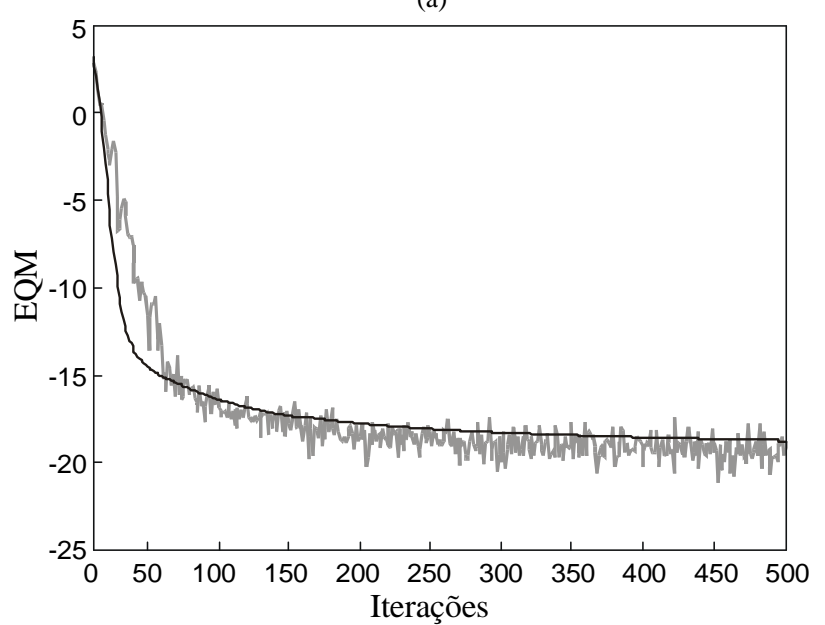

(b)

Fig. 5. Exemplo 1. Sinal de entrada correlacionado. (a) Comportamento médio do vetor de coeficientes: (linha cinza) simulação MC; (linha preta) modelo proposto. (b) Curva de aprendizagem: (linha irregular cinza) simulação MC; (linha contínua preta) modelo proposto.

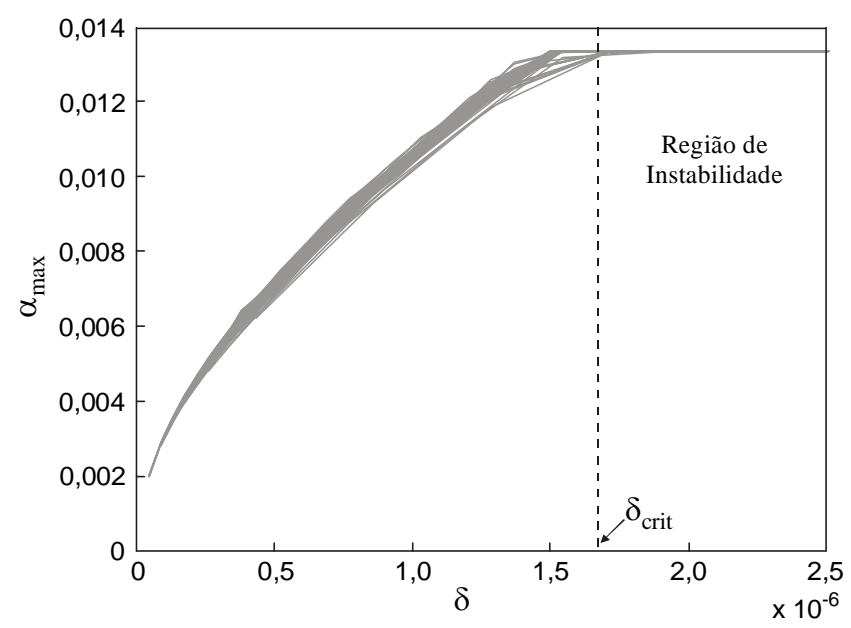

Fig. 6. Variação de $\alpha_{\max }$ em função de $\delta=0,5 \alpha_{\mathrm{f}} \beta \gamma$ para um filtro de 50 coeficientes.

\section{CONCLUSÕES}

Neste trabalho, são apresentadas novas expressões analíticas para descrever o comportamento do algoritmo NCLMS. Também, são realizadas algumas considerações relativas à robustez e estabilidade do algoritmo em relação ao valor dos parâmetros de controle.

\section{REFERÊNCIAS}

[1] S. Haykin, Adaptive Filter Theory, $4^{\text {th }}$ ed., Upper Saddle River, NJ: Prentice Hall, 2002.

[2] R. H. Kwong and E. W. Johnston, “A variable step size LMS algorithm,” IEEE Trans. Signal Process., vol. 40, no. 7, pp. 1633-1642, Jul. 1992.

[3] B. Farhang-Boroujeny, "Variable step size LMS algorithm - New developments and experiments," IEE Proceedings - Vision, Image, Signal Process., vol. 141, no. 5, pp. 311-317, Oct. 1994.

[4] Y. Wei and S. B. Gelfand, "Noise-constrained least mean squares algorithm,” IEEE Trans. Signal Process., vol. 49, no. 9, pp. 1961-1970, Sept 2001.

[5] P. Kuosmanen, R. Bilcu, and C. Rusu, "A noise constrained VS-LMS algorithm,” in Proc. IEEE/AFCEA Eurocomm 2000 Information Systems for Enhanced Public Safety and Security, Munich, Germany, May 2000, pp. 29-33.

[6] H. Robbins and S. Monro, “A stochastic approximation method,” Annals of Mathematical Statistics, vol. 22, no. 3, pp. 400-407, Sept. 1951.

[7] N. J. Bershad, P. Celka, and J. M. Vesin, "Stochastic analysis of gradient adaptive identification of nonlinear systems with memory for Gaussian data and noisy input and output measurements,” IEEE Trans. Signal Process., vol. 47, no. 3, pp. 675-689, Mar. 1999.

[8] S. B. Gelfand, Y. Wei, and J. V. Krogmeier, "The stability of variable step-size LMS algorithms,” IEEE Trans. Signal Process., vol. 47, no. 12, pp. 3277-3288, Dec. 1999.

[9] T. Yang and B. Shahrrava, "Performance of variable step-size LMS algorithms for linear adaptive inverse control systems," in Proc. IEEE Canadian Conf. on Electrical and Computer Engineering, Saskatoon, Canada, May 2005, pp. 755-758. 